\title{
Useful resources
}

UK

Bloodwise

Tel: +44 (0)207 5042200

Support: 08082080888

www.bloodwise.org.uk

\section{Cancer Research UK}

Tel: 08088004040

supporter.services@cancer.org.uk

www.cancerresearchuk.org

\section{Leuka}

Tel: +44 (0)20 72990722

info@leuka.org.uk

www.leuka.org.uk

\section{Leukaemia Cancer Society}

Tel: +44 (0)20 83744821

info@leukaemiacancersociety.org

www.leukaemiacancersociety.org

\section{Leukaemia Care}

Tel: +44 (0)1905 755977

Careline: 08088010444

support@leukaemiacare.org.uk

www.leukaemiacare.org.uk

Leukaemia \& Myeloma Research UK

Tel: +44 (0)1515450800

cs@leukaemiamyelomaresearchuk.org

www.lmruk.org

\section{Leukaemia UK}

Tel: +44 (0)20 79227982

contribute@leukaemiauk.org.uk

www.leukaemiauk.org.uk
USA

American Cancer Society

Toll-free: 18002272345

www.cancer.org

Cancer Treatment Centers of America

Tel: +1 8554089992

www.cancercenter.com/cancer-types/

leukemia

Leukemia Research Foundation

Tel: +1 8474240600

www.allbloodcancers.org

Leukemia \& Lymphoma Society

Toll-free: 18009554572

www.lls.org

International

Cancer Association of South Africa

Toll-free: 0800226622

www.cansa.org.za

Leukaemia and Blood Cancer

New Zealand

Toll-free: 0800151015

Tel: +64 (0)9 6383556

www.leukaemia.org.nz

Leukaemia Foundation (Australia)

Toll-free 1800620420

info@leukaemia.org.au

www.leukaemia.org.au

Leukemia and Lymphoma Society

of Canada

Tel: +1 8332224884

AdminCanada@lls.org

www.llscanada.org 